\title{
Continuous Renal Replacement Therapy Is Associated With Reduced Serum Ammonia Levels and Mortality in Acute Liver Failure
}

Filipe S. Cardoso, ${ }^{1}$ Michelle Gottfried, ${ }^{2}$ Shannan Tujios, ${ }^{3}$ Jody C. Olson, ${ }^{4}$ and Constantine J. Karvellas $\mathbb{D},{ }^{5}$ For the US Acute Liver Failure Study Group

Hyperammonemia has been associated with intracranial hypertension and mortality in patients with acute liver failure (ALF). We evaluated the effect of renal replacement therapy (RRT) on serum ammonia level and outcomes in ALF. This was a multicenter cohort study of consecutive ALF patients from the United States ALF Study Group registry between January 1998 and December 2016. First, we studied the association of ammonia with hepatic encephalopathy (HE) and 21-day transplant-free survival (TFS; $n=1,186$ ). Second, we studied the effect of RRT on ammonia for the first 3 days post study admission $(n=340)$ and on 21 -day TFS $(n=1,186)$. Higher admission $(n=1,186)$ median ammonia level was associated with grade 3-4 HE (116 vs. $83 \mu \mathrm{mol} / \mathrm{L})$ and mortality at day 21 attributed to neurological (181 vs. 90 $\mu \mathrm{mol} / \mathrm{L}$ ) and all causes (114 vs. $83 \mu \mathrm{mol} / \mathrm{L} ; P<0.001$ for all). Among 340 patients with serial ammonia levels, 61 (18\%) were on continuous RRT (CRRT), 59 (17\%) were on intermittent RRT (IRRT), and 220 (65\%) received no RRT for the first 2 days. From days 1 to 3, median ammonia decreased by 38\%, 23\%, and 19\% with CRRT, IRRT, and no RRT, respectively. Comparing to no RRT use, whereas ammonia reduction with CRRT was significant $(P=0.007)$, with IRRT it was not $(P=0.75)$. After adjusting for year of enrollment, age, etiology, and disease severity, whereas CRRT (odds ratio [OR], 0.47 [95\% confidence interval $\{\mathrm{CI}\}, 0.26-0.82]$ ) was associated with reduction in 21-day transplant-free all-cause mortality, IRRT (OR, 1.68 [95\% CI, 1.04-2.72]) was associated with an increase. Conclusion: In a large cohort of ALF patients, hyperammonemia was associated with high-grade HE and worse 21-day TFS. CRRT was associated with a reduction in serum ammonia level and improvement of 21-day TFS. (HePATOLOGY 2018;67:711-720).

$\mathrm{I}$ $\mathrm{n}$ acute liver failure (ALF), hepatocyte loss results in reduced ammonia detoxification and hyperammonemia, both through reductions in urea cycle (conversion to urea) and glutamine metabolism. With the frequent coexistent acute kidney injury in ALF, reduced renal ammonia excretion further worsens hyperammonemia. ${ }^{(1)}$

In ALF, hyperammonemia has been associated with hepatic encephalopathy ( $\mathrm{HE})$, cerebral edema, intracranial hypertension, and mortality from cerebral herniation. ${ }^{(2,3)}$ The mechanisms by which ammonia causes this neurological dysfunction are still not fully understood. Whether by direct effect or after

Abbreviations: ALF, acute liver failure; ALT, alanine aminotransferase; APAP, acetaminophen; $A U C$, area under the receiver operating characteristic curve; CI, confidence interval; CRRT, continuous renal replacement therapy; HE, hepatic encephalopathy; IMV, invasive mechanical ventilation; $I N R$, international normalized ratio; IRRT, intermittent renal replacement therapy; IQR, interquartile range; OLT, orthotopic liver transplantation; $O P A$, ornithine phenylacetate; $a O R$, adjusted odds ratio; OR, odds ratio; RRT, renal replacement therapy; TFS, transplant-free survival; US-ALFSG, United States Acute Liver Failure Study Group.

Received June 12, 2017; accepted August 23, 2017.

Additional Supporting Information may be found at onlinelibrary.wiley.com/doi/10.1002/hep.29488/suppinfo.

Sponsored by NIH grant U-01 58369 (from NIDDK).

Members and institutions participating in the US Acute Liver Failure Study Group are listed in the Appendix.

This article followed the STROBE guideline for reporting cohort studies (BMJ, 2007).

Copyright (C) 2017 by the American Association for the Study of Liver Diseases.

View this article online at wileyonlinelibrary.com.

DOI 10.1002/hep. 29488

Potential conflict of interest: Nothing to report. 
metabolization to glutamine in the astrocytes, ammonia may contribute to local altered neurotransmitter action, impaired mitochondrial function, and increased oxidative stress, which may lead to downstream astrocytic swelling and cerebral edema. ${ }^{(4,5)}$

In ALF, current ammonia-lowering strategies rely mainly on supportive treatment for organ failures. ${ }^{(6)}$ However, the use of extracorporeal devices has been proposed to reduce serum ammonia level. ${ }^{(7,8)}$ Recently, in a small cohort study, patients with acute or chronic liver dysfunction and hyperammonemia on renal replacement therapy (RRT) for 24 hours showed a significant reduction in serum ammonia level. ${ }^{(9)}$

To date, no formal study has evaluated RRT effect on serum ammonia level in patients with ALF. In this context, we hypothesized that RRT use would significantly reduce serum ammonia level in comparison to no RRT support. Therefore, we aimed to: determine the association of serum ammonia level and patients' clinical outcomes; evaluate the effect of RRT on serum ammonia level throughout a 3-day period; and assess the effect of RRT on patients' survival.

\section{Patients and Methods}

\section{DESIGN, SETTING, AND PARTICIPANTS}

We performed a retrospective cohort study of patients with ALF enrolled in the United States ALF Study Group (US-ALFSG) prospective registry between January 1998 and August 2016. Patients not fulfilling criteria for ALF (see operational definitions) or with lack of data on serum ammonia level or RRT status at study admission were initially excluded (Supporting Fig. S1; original cohort, $n=1,186$ ). For the analysis of ammonia dynamics between days 1 and 3 post study admission, patients lacking data on serum ammonia level or RRT status for either of these days were excluded ( $n=340)$. Within this subset of patients, a subanalysis was performed following exclusion of the 16 patients enrolled as well in the USALFSG STOP-ALF trial designed to determine safety and tolerability of ornithine phenylacetate (OPA), a putative ammonia lowering agent, given that the use of OPA might confound the results.

The study's protocol has been approved by the institutional review board or health research ethics board at all enrolling sites (tertiary liver transplantation referral centers) within the US-ALFSG (see Appendix). Informed consent was obtained from next of kin for all enrolled patients because were unable to provide it on their own because of $\mathrm{HE}$. All research procedures were conducted according to the principles of the Declaration of Helsinki. ${ }^{(10)}$

\section{OPERATIONAL DEFINITIONS}

ALF was defined using the following criteria: (1) HE of any degree; (2) international normalized ratio (INR) $\geq 1.5$; (3) acute illness onset $<26$ weeks; and (4) no evidence of cirrhosis. Given the reported similar clinical impact, we considered both arterial $(n=509$ at study admission) and venous ( $n=677$ at study admission) ammonia levels. ${ }^{(11)}$ RRT included intermittent (IRRT), such as hemodialysis or sustained low-efficiency dialysis, and continuous (CRRT) modalities. ${ }^{(12)}$ To avoid understating IRRT efficiency and facilitate comparisons with CRRT, the analysis of ammonia dynamics between days 1 and 3 post study admission considered RRT use on days 1 and 2 (up to 48 hours). The use of RRT within the US-ALFSG is not protocolized; therefore, indications, modality, replacement fluid, anticoagulation, treatment dose, and

\section{ARTICLE INFORMATION:}

From the ${ }^{1}$ Intensive Care Unit, Curry Cabral Hospital, Central Lisbon Hospital Center, Lisbon, Portugal; ${ }^{2}$ Department of Public Health Sciences, Medical University of South Carolina, Charleston, SC; ${ }^{3}$ Division of Digestive and Liver Diseases, University of Texas Southwestern Medical Center, Dallas, TX; ${ }^{4}$ Department of Internal Medicine, University of Kansas Medical Center, Kansas City, KS; and ${ }^{5}$ Departments of Critical Care and Division of Gastroenterology (Liver Unit), University of Alberta, Edmonton, Alberta, Canada.

\section{ADDRESS CORRESPONDENCE AND REPRINT REQUESTS TO:}

Constantine J. Karvellas, M.D., S.M., F.R.C.P.C.

Division of Gastroenterology (Liver Unit), Department of Critical Care

Medicine, University of Alberta

1-40 Zeidler Ledcor Building
Edmonton, Alberta, Canada, T6G-2X8

E-mail: dean.karvellas@ualberta.ca

Tel: +1-780-248-1555 
timings for initiation and suspension of RRT were based on clinical judgment at each enrolling site.

\section{EXPOSURES AND ENDPOINTS}

The US-ALFSG registry data repository (Medical University of South Carolina, Charleston, SC) contains demographic, clinical, and outcome data. Data were retrieved on the following baseline characteristics of patients with ALF: age; sex; race; etiology; HE (West Haven criteria); organ support requirements (invasive mechanical ventilation [IMV], vasopressors, and RRT); laboratory serum profile (ammonia, INR, bilirubin, alanine aminotransferase [ALT], creatinine, potassium, phosphate, $\mathrm{pH}$, and lactate); nacetylcysteine use; and clinical outcomes (21-day post study admission transplant-free survival [TFS], overall survival, and orthotopic liver transplantation [OLT]).

The primary endpoint for this study was the difference in serum ammonia level between days 1 and 3 post study admission. The secondary endpoint was $21-$ day post study admission TFS.

Based on previous literature, we could expect a reduction of median serum ammonia level of $22 \%$ after 24 hours of CRRT. ${ }^{(9)}$ Accordingly, to detect a difference of $15 \%$ in median serum ammonia level between RRT and no RRT use, with $90 \%$ power and a cutoff for statistical significance of 0.05 , we would require a sample with at least 110 patients in each arm. ${ }^{(13)}$

\section{STATISTICAL ANALYSIS}

Categorical variables were presented as proportions and continuous variables as median and interquartile ranges (IQRs). For the baseline characteristics, comparisons for independent samples were performed with chi-square test or Mann-Whitney test. For the serial ammonia analysis, comparisons for related samples were done with the Friedman test. For the baseline characteristics and serial ammonia analyses, missing data were $3 \%$ and $5 \%$, respectively; therefore, no multiple imputations were performed.

The study of associations with 21-day post study admission transplant-free all-cause mortality was done with logistic regression. For multivariate analysis, covariates were initially chosen based on clinical relevance or $P<0.10$ on univariate comparisons, including: year of admission, age, etiology, HE, IMV, vasopressors, RRT, ammonia, bilirubin, creatinine, potassium, phosphate, $\mathrm{pH}$, lactate, and n-acetylcysteine. Final models were derived based on a step-wise backward elimination process. Collinearity was evaluated and avoided where appropriate. Models' performance was assessed by chi-square test and area under the receiver operating characteristic curve (AUC). All comparisons considered a threshold for statistical significance of 0.05. Statistical analysis was performed using IBM SPSS Statistics (version 20; IBM Corp, North Castle, NY).

\section{Results}

\section{PATIENTS' BASELINE CHARACTERISTICS}

A total of 1,186 consecutive patients with ALF and complete data on ammonia level and RRT status at study admission were identified within the USALFSG registry between January 1998 and August 2016 (Supporting Fig. S1).

Median (IQR) age was 39 (29-52) years, and 819 patients (69\%) were females. Acetaminophen (APAP)-related ALF occurred in 593 (50\%) patients. All etiologies are specified in Supporting Fig. S2.

At study admission, 600 (51\%) patients developed grade 3-4 HE. IMV, vasopressors, and RRT were required in 632 (56\%), 299 (25\%), and $314(26 \%)$ patients, respectively. Median (IQR) INR, ammonia, creatinine, and lactate levels were 2.8 (2.1-4.2), 97 (63-157) $\mu \mathrm{mol} / \mathrm{L}, 1.70(0.90-3.16) \mathrm{mg} / \mathrm{dL}$, and 4.1 (2.4-7.7) $\mathrm{mmol} / \mathrm{L}$, respectively. All baseline characteristics of the original cohort are depicted in Table 1.

\section{ASSOCIATION OF SERUM AMMONIA LEVEL WITH PATIENTS' CLINICAL OUTCOMES}

In the original cohort, 1,051 patients had complete data on both OLT (1,169 patients if OLT status only) and survival (1,067 patients if survival status only) status at 21 days post study admission. Among them, 224 (21\%) were transplanted and 493 (60\% of nontransplanted patients) survived without OLT at 21 days post study admission (Table 1). The causes of mortality are specified in Supporting Fig. S3.

Median ammonia level at study admission was significantly greater for patients with grade 3-4 versus grade $1-2 \mathrm{HE}$ (116 vs. $83 \mu \mathrm{mol} / \mathrm{L}$ ) or who died from neurological cause versus all others (181 vs. $90 \mu \mathrm{mol} /$ $\mathrm{L})$ or any cause versus alive (114 vs. $83 \mu \mathrm{mol} / \mathrm{L})$ at 21 
TABLE 1. Baseline Characteristics of Patients With ALF at Study Admission $(n=1,186)$

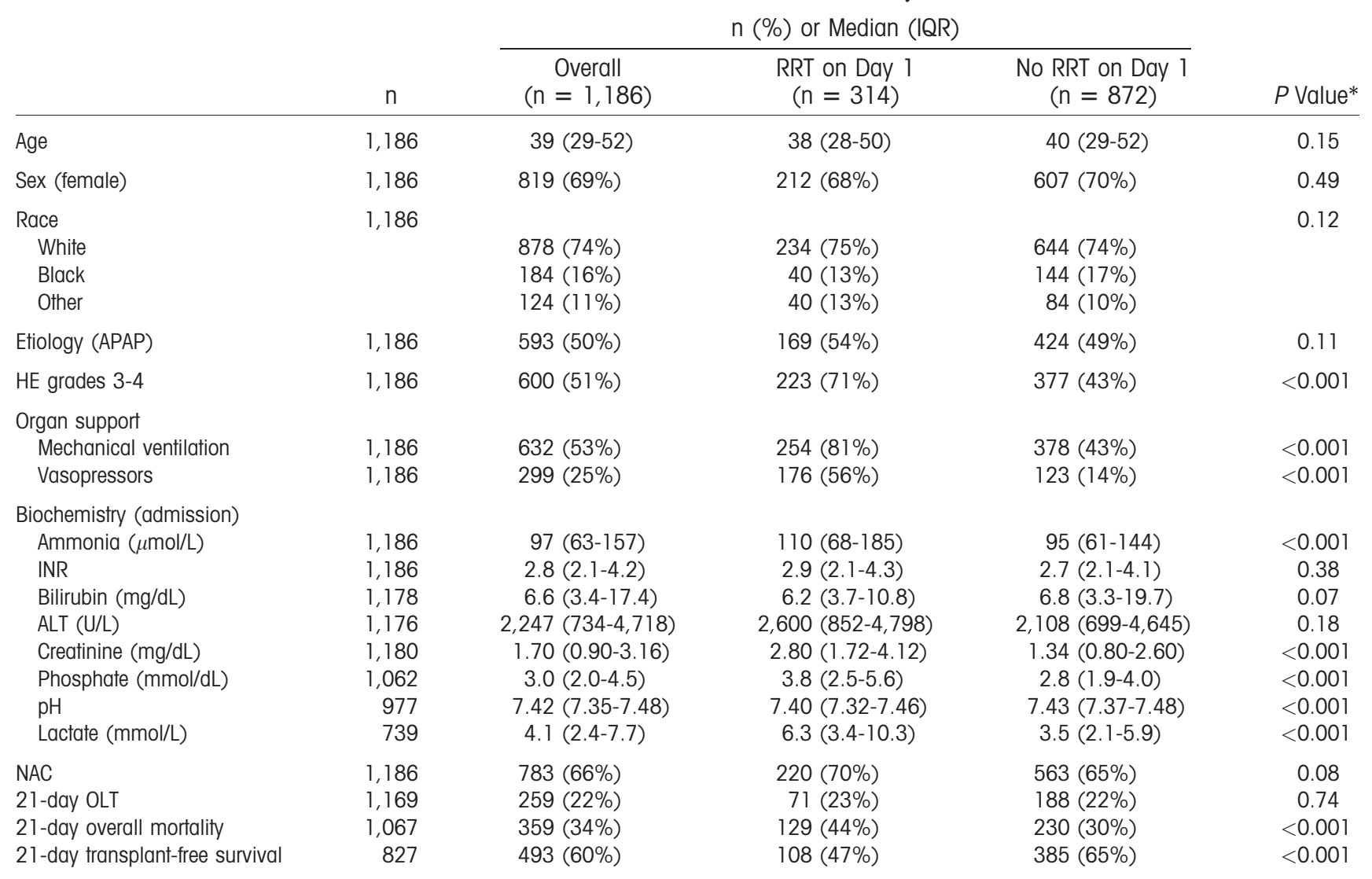

*Mann-Whitney U or chi-square tests. $\alpha=0.05$.

Abbreviation: NAC, $N$-acetylcysteine.

days post study admission (Table $2 ; P<0.001$ for all comparisons).

The ammonia cutoffs $\geq 100, \geq 150$, and $\geq 200$ $\mu \mathrm{mol} / \mathrm{L}$ at study admission were associated with 21 day neurological mortality (without OLT) with a sensitivity of $77 \%, 58 \%$, and $42 \%$ respectively, and a specificity of $55 \%, 76 \%$, and $87 \%$, respectively (AUC of $0.72[95 \%$ confidence interval $\{\mathrm{CI}\}, 0.65-0.79]$ ).

\section{ASSOCIATION OF PATIENTS' BASELINE CHARACTERISTICS WITH RRT REQUIREMENT}

Patients on RRT at study admission had significantly greater proportions of grade $3-4 \mathrm{HE} \mathrm{(71 \%} \mathrm{vs.}$ $43 \%)$ and requirement for IMV (81\% vs. $43 \%)$ and vasopressors (56\% vs. $14 \%$; $P<0.001$ for all comparisons). These patients had also significantly greater admission median ammonia (110 vs. $95 \mu \mathrm{mol} / \mathrm{L})$, creatinine $(2.80$ vs. $1.34 \mathrm{mg} / \mathrm{dL})$, phosphate (3.8 vs. 2.8 $\mathrm{mmol} / \mathrm{dL}$ ), and lactate (6.3 vs. $3.5 \mathrm{mmol} / \mathrm{L}$ ) levels ( $P<0.001$ for all comparisons). Furthermore, they had significantly lower 21-day post study admission TFS (47\% vs. $65 \% ; P<0.001$ ). Stratification of all baseline characteristics of the original cohort by RRT requirement at study admission is depicted in Table 1.

Patients admitted during the 2007-2016 period were significantly more likely to be on RRT on day 1 than the ones admitted in the 1998-2006 period (62\% vs. $38 \% ; P=0.005)$. At study admission, being on IRRT was significantly more frequent in the 19982006 period ( $48 \%$ vs. $52 \% ; P=0.027$ ), whereas being on CRRT was more common in the 2007-2016 period (83\% vs. $17 \% ; P<0.001$ ).

Among patients on RRT at study admission ( $\mathrm{n}=$ 314), the ones on CRRT ( $n=119)$ had significantly greater proportion of requirement for vasopressors (64\% vs. $51 \% ; P=0.029)$ than the ones on IRRT $(n=195)$. Patients on CRRT had also significantly greater admission median INR (3.2 vs. $2.7 ; P=$ 
TABLE 2. Association of Ammonia Level With Clinical Outcomes of Patients With ALF $(\mathbf{n}=1,186)$

\begin{tabular}{|c|c|c|c|c|}
\hline & $\begin{array}{r}\text { Ammonia ( } \boldsymbol{\mu} \\
{[\mathrm{me}}\end{array}$ & $=1,186)$ & OR $(95 \% \mathrm{Cl})^{*}$ & $P$ Value \\
\hline & Yes & No & & \\
\hline $\begin{array}{l}\text { HE grades } 3-4 \text { at study admission } \\
(n=1,186)\end{array}$ & $116(77-186)$ & $83(54-123)$ & $1.006(1.005-1.008)$ & $<0.001$ \\
\hline $\begin{array}{l}\text { 21-day transplant-free neurological mortality } \\
(n=613)\end{array}$ & $181(102-257)$ & $90(57-146)$ & $1.004(1.003-1.006)$ & $<0.001$ \\
\hline 21-day transplant-free all-cause mortality & $114(68-184)$ & $83(52-124)$ & $1.005(1.003-1.006)$ & $<0.001$ \\
\hline
\end{tabular}

$(n=827)$

$\alpha=0.05$

*Per unit increment.

${ }^{\dagger}$ Logistic regression.

$0.003)$ and lower admission median creatinine (2.3 vs. $3.2 \mathrm{mg} / \mathrm{dL} ; P<0.001)$, phosphate $(4.2$ vs. $3.3 \mathrm{mmol} /$ $\mathrm{dL} ; P=0.034)$, and lactate $(6.2$ vs. $7.9 \mathrm{mmol} / \mathrm{L} ; P=$ $0.025)$ levels. However, there was no significant difference on admission median ammonia level (133 vs. $102 \mu \mathrm{mol} / \mathrm{L} ; P=0.052)$. All baseline characteristics of patients on RRT at study admission stratified by type of RRT (CRRT vs. IRRT) are depicted in Supporting Table S1.

\section{SERUM AMMONIA DYNAMICS BETWEEN DAYS 1 AND 3 POST STUDY ADMISSION}

A total of 340 patients with ALF and complete data on ammonia level and RRT status between days 1 and
3 post study admission were identified within the USALFSG registry between January 1998 and August 2016 (Supporting Fig. S1).

Between days 1 and 3 post study admission, a maximum of 63 (19\%), 56 (16\%), and 221 (65\%) patients were on IRRT, CRRT, or no RRT at all at any day, respectively. During this 3-day period, median (day 1 vs. day 3 ) ammonia (100 vs. $81 \mu \mathrm{mol} / \mathrm{L})$, INR (3.0 vs. $2.0)$, creatinine (1.50 vs. $1.49 \mathrm{mg} / \mathrm{dL})$, lactate (4.2 vs. $2.8 \mathrm{mmol} / \mathrm{L}$ ), and bilirubin (5.3 vs. $7.8 \mathrm{mg} / \mathrm{dL}$ ) levels varied significantly $(P<0.001$ for all day 1 vs. day 2 vs. day 3 comparisons). Patients' RRT status and biochemical changes between days 1 and 3 post study admission are depicted in Table 3.

Between days 1 and 3 post study admission, the reduction of median ammonia level differed with the

TABLE 3. Serial Characteristics of Patients With ALF From Days 1 to 3 Post Study Admission $(\mathbf{n}=340)$

\begin{tabular}{|c|c|c|c|c|c|c|c|}
\hline & $\mathrm{n}$ & Day 1 & $\mathrm{n}$ & Day 2 & $\mathrm{n}$ & Day 3 & $P$ Value* \\
\hline \multicolumn{8}{|l|}{ RRT (on each day) } \\
\hline Overall & 340 & 91 (27\%) & 340 & 112 (33\%) & 340 & $119(35 \%)$ & $<0.001$ \\
\hline CRRT & 340 & 45 (13\%) & 340 & $56(17 \%)$ & 340 & $56(17 \%)$ & 0.028 \\
\hline IRRT & 340 & $46(14 \%)$ & 340 & $56(17 \%)$ & 340 & $63(19 \%)$ & 0.001 \\
\hline HE grades $3-4$ & 340 & $170(50 \%)$ & 321 & 179 (56\%) & 340 & $173(51 \%)$ & 0.029 \\
\hline \multicolumn{8}{|l|}{ Ammonia $(\mu \mathrm{mol} / \mathrm{L})$} \\
\hline Overall & 340 & $100(70-157)$ & 340 & 88 (64-127) & 340 & $81(52-112)$ & $<0.001$ \\
\hline $\begin{array}{l}\text { CRRT } \\
\text { (on days 1-2) }\end{array}$ & 61 & 140 (79-192) & 61 & $103(78-172)$ & 61 & $87(66-125)$ & $<0.001$ \\
\hline $\begin{array}{l}\text { IRRT } \\
\text { (on days 1-2) }\end{array}$ & 59 & $98(69-133)$ & 59 & $89(63-130)$ & 59 & $75(44-116)$ & 0.066 \\
\hline $\begin{array}{l}\text { No RRT } \\
\text { (on days 1-2) }\end{array}$ & 220 & $97(68-145)$ & 220 & $82(61-119)$ & 220 & $79(50-105)$ & $<0.001$ \\
\hline INR & 340 & $3.0(2.1-4.3)$ & 336 & $2.4(1.8-3.4)$ & 331 & $2.0(1.6-3.0)$ & $<0.001$ \\
\hline Bilirubin (mg/dL) & 337 & $5.3(3.0-15.4)$ & 333 & $6.3(3.4-14.4)$ & 332 & $7.8(4.0-16.5)$ & $<0.001$ \\
\hline Creatinine $(\mathrm{mg} / \mathrm{dL})$ & 337 & $1.50(0.90-2.80)$ & 340 & $1.54(0.80-2.79)$ & 336 & $1.49(0.80-2.44)$ & $<0.001$ \\
\hline Lactate (mmol/L) & 242 & $4.2(2.5-8.0)$ & 193 & $3.1(2.0-6.1)$ & 166 & $2.8(1.7-5.8)$ & $<0.001$ \\
\hline
\end{tabular}

$\alpha=0.05$

*Friedman test (comparisons of day 1 vs. day 2 vs. day 3 ). 

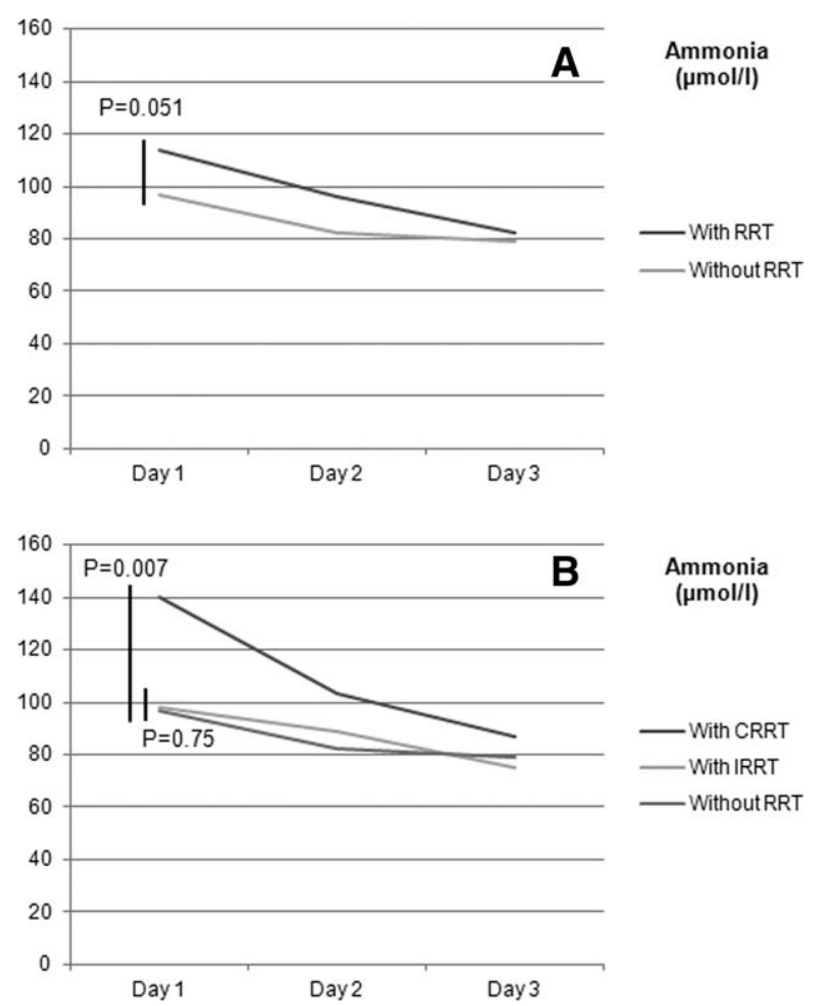

FIG. 1. (A,B) Renal replacement therapy on days 1 and 2 and median serum ammonia level $(\mu \mathrm{mol} / \mathrm{L})$ between days 1 and 3 post study admission $(\mathrm{n}=340)$.

type of RRT used during days 1 and 2 (Fig. 1): 28.1\% with any type of RRT (120 patients); 23.4\% with IRRT (any of days 1 and 2 but without CRRT - 59 patients); 37.9\% with CRRT (any of days 1 and 2 with or without IRRT-61 patients); and 18.6\% without any RRT at all (220 patients). Whereas median ammonia level reduction with CRRT was significantly greater than without RRT use $(P=0.007)$, with IRRT it was not $(P=0.75)$.

The variations in ammonia dynamics between days 1 and 3 post study admission with each type of RRT stratified by etiology of ALF (APAP vs. other etiologies) are depicted in Supporting Table S2. Ammonialevel reduction with CRRT was greater than no RRT use for both etiology subgroups (38.6\% vs. $21.6 \%$ for APAP and $38.5 \%$ vs. $11.3 \%$ other etiologies), but did not reach statistical significance probably attributed to lower number of patients in each treatment arm $(P=$ 0.085 and $P=0.054$, respectively).

Ammonia dynamics between days 1 and 3 post study admission were similar within the subgroup of patients with ALF following exclusion of the 16 patients included in the US-ALFSG trial on the impact of OPA for HE management in ALF (Supporting Table S3). Therefore, the overall results were not affected by OPA therapy.

\section{ASSOCIATION OF RRT WITH PATIENTS' SURVIVAL}

We derived three models to better assess the effect of RRT (overall, IRRT, or CRRT) on 21-day post study admission transplant-free all-cause mortality (Table 4). After adjusting for year of admission (20072016 vs. 1998-2006), age, etiology (APAP vs. nonAPAP), and disease severity (requirement for IMV, vasopressors, INR, creatinine, and ammonia levels), RRT use on day 1 was significantly associated with lower 21-day post study admission transplant-free allcause mortality (adjusted odds ratio [aOR] of 0.47 [95\% CI, 0.26-0.82]). Conversely, IRRT use on day 1 was significantly associated with greater 21 -day post study admission transplant-free all-cause mortality (aOR of 1.68 [95\% CI, 1.04-2.72]). The performance of all 3 models was good as defined by AUC values greater than 0.80 .

\section{Discussion}

\section{KEY RESULTS AND COMPARISONS WITH PREVIOUS STUDIES}

Using a large cohort of patients with ALF, we have shown that the degree of hyperammonemia was significantly associated with worse clinical outcomes, including high-grade (3-4) HE and lower 21-day post study admission TFS $(P<0.001$ for both comparisons). These findings are consistent with previous reports suggesting the use of hyperammonemia as a severity marker in $\mathrm{ALF}^{(14,15)}$

Patients with ALF on RRT at study admission had greater disease severity, especially in terms of extrahepatic organ failures: More patients demonstrated highgrade $\mathrm{HE}$ and requirement for IMV and vasopressors, as well as greater median ammonia, creatinine, and lactate levels $(P<0.001$ for all comparisons). Furthermore, these patients had lower 21-day post study admission TFS (47\% vs. $65 \% ; P<0.001$ ). These findings likely reflect the choice of RRT for patients with the most severe clinical course of ALF, including acute 
TABLE 4. Association of Renal Replacement Therapy Use on Day 1 With 21-Day Post Study Admission Transplant-Free All-Cause Mortality for Patients With ALF $(n=1,186)$

\begin{tabular}{|c|c|c|c|c|c|c|}
\hline & \multicolumn{2}{|l|}{ Model $\rceil^{*}$} & \multicolumn{2}{|l|}{ Model $2^{\dagger}$} & \multicolumn{2}{|l|}{ Model $3^{\ddagger}$} \\
\hline & aOR $(95 \% \mathrm{Cl})$ & $P$ Value $^{\S}$ & aOR $(95 \% \mathrm{Cl})$ & $P$ Value $^{\S}$ & aOR $(95 \% \mathrm{Cl})$ & $P$ Value $^{\S}$ \\
\hline $\begin{array}{l}\text { Year of admission (2007-2016 } \\
\text { vs. 1998-2006) }\end{array}$ & $0.48(0.34-0.68)$ & $<0.001$ & $0.49(0.34-0.69)$ & $<0.001$ & $0.52(0.37-0.75)$ & $<0.001$ \\
\hline Age & $1.04(1.03-1.05)$ & $<0.001$ & $1.04(1.03-1.05)$ & $<0.001$ & $1.04(1.02-1.05)$ & $<0.001$ \\
\hline APAP & $0.28(0.20-0.41)$ & $<0.001$ & $0.28(0.20-0.40)$ & $<0.001$ & $0.27(0.19-0.40)$ & $<0.001$ \\
\hline IMV & $2.34(1.60-3.42)$ & $<0.001$ & $2.25(1.54-3.29)$ & $<0.001$ & $2.41(1.65-3.52)$ & $<0.001$ \\
\hline Vasopressors & $3.67(2.38-5.67)$ & $<0.001$ & $3.40(2.25-5.12)$ & $<0.001$ & $4.34(2.82-6.69)$ & $<0.001$ \\
\hline INR & $1.28(1.17-1.40)$ & $<0.001$ & $1.29(1.18-1.41)$ & $<0.001$ & $1.28(1.18-1.40)$ & $<0.001$ \\
\hline Creatinine (mg/dL) & $1.10(1.00-1.21)$ & 0.041 & $1.07(0.97-1.18)$ & 0.16 & $1.11(1.01-1.21)$ & 0.032 \\
\hline Ammonia $(\mu \mathrm{mol} / \mathrm{L})$ & $1.004(1.002-1.006)$ & $<0.001$ & $1.004(1.002-1.006)$ & $<0.001$ & $1.004(1.002-1.006)$ & $<0.001$ \\
\hline RRT & $0.99(0.64-1.51)$ & 0.95 & NA & NA & NA & NA \\
\hline IRRT & NA & NA & $1.68(1.04-2.72)$ & 0.035 & NA & NA \\
\hline CRRT & NA & NA & NA & NA & $0.47(0.26-0.82)$ & 0.008 \\
\hline
\end{tabular}

$\alpha=0.05$.

${ }^{*}$ Model 1: $\mathrm{n}=822,21$-day spontaneous mortality $=334$, chi-square test $=273$, AUC of 0.82 (95\% CI, 0.79-0.85).

${ }^{\dagger}$ Model 2: $\mathrm{n}=822,21$-day spontaneous mortality $=334$, chi-square test $=278$, AUC of $0.82(95 \% \mathrm{CI}, 0.79-0.85)$.

${ }^{\ddagger}$ Model 3: $\mathrm{n}=822,21$-day spontaneous mortality $=334$, chi-square test $=281$, AUC of $0.82(95 \% \mathrm{CI}, 0.80-0.85)$.

${ }^{\S}$ Logistic regression.

Abbreviation: NA, not applicable.

kidney injury and metabolic acidosis, the most common clinical context for RRT initiation. ${ }^{(2,16,17)}$

In addition, RRT use in patients with ALF increased greatly between the 1998-2006 and 20072016 periods ( $38 \%$ vs. $62 \% ; P=0.005)$, especially CRRT utilization $(17 \%$ vs. $83 \% ; P<0.001)$. These findings likely reflect the increasing use of RRT in clinical practice to treat acute kidney injury in general patients, including those with liver dysfunction. Previous studies have shown a significant improvement in survival and renal function recovery with the timely use of RRT, especially in the most severe cases of acute kidney injury. ${ }^{(18,19)}$

We have also found that RRT use on days 1 and 2 post study admission was associated with a trend toward reduced median serum ammonia level between days 1 and 3 post study admission when compared with no RRT utilization $(28.1 \%$ vs. $18.6 \%$; $P=$ 0.051). Whereas such a reduction was significant with CRRT (37.9\% vs. $18.6 \%$; $P=0.007)$, with IRRT it was not $(23.4 \%$ vs. $18.6 \% ; P=0.75)$.

This study provides evidence of CRRT capacity to reduce serum ammonia level in ALF in comparison with IRRT and no RRT use. In a small cohort study including patients with acute or chronic liver dysfunction and hyperammonemia $(\mathrm{n}=24)$, Slack et al. reported an overall reduction of $22 \%$ on median serum ammonia level with 24 hours' use of CRRT. ${ }^{(9)}$ In our cohort, the overall reduction in median serum ammonia level with CRRT was greater (37.9\%). This difference may be partially explained by the type of patients included in our cohort (only ALF) and the longer course of CRRT utilized (up to 48 hours after study admission). Furthermore, other factors have been described to contribute to CRRT efficiency in this context, for example: $\mathrm{pH}$, blood and replacement fluid flow rates, filter surface area and clotting, and treatment interruptions. ${ }^{(7,9,20)}$ Median $\mathrm{pH}$ was found to be within normal range in patients with and without RRT at study admission (7.40 vs. $7.43 ; P<0.001$ ), therefore it unlikely affected ammonia dynamics. However, data regarding other such technical aspects of RRT were not recorded in the US-ALFSG registry (see Limitations).

Finally, we showed that CRRT use at day 1 was independently associated (after adjusting for year of admission, age, etiology, and disease severity) with lower 21-day post study admission transplant-free allcause mortality (aOR of 0.47 ). Conversely, IRRT was independently associated with higher 21-day post study admission transplant-free all-cause mortality (aOR of 1.68).

In a study designed to study the impact of highvolume plasma exchange on hospital survival of patients with ALF, Larsen et al. reported that median serum ammonia levels decreased significantly more rapidly with high-volume plasma exchange than with standard medical treatment $(29.7 \%$ vs. $1.5 \%$ between days 1 and 3 post study admission). ${ }^{(21)}$ Furthermore, high-volume plasma exchange significantly improved hospital TFS (58.7\% vs. $47.8 \%$ ). The plasma exchange protocol used in this study consisted of replacing 8-12 
$\mathrm{L}$ of plasma per day (at 1-2 L per-hour rate) for 3 days using $100 \%$ fresh frozen plasma. Whereas this technique may resemble a subtype of IRRT, the composition of the replacement fluid is different from the ones used in regular RRT sessions. Moreover, plasma exchange efficacy may, in part, be attributed to a specific dampening effect on the innate immune system response.

Previous studies have evaluated the effect of RRT on hemodynamics in patients with ALF and acute kidney injury. They have shown that, whereas IRRT use has been associated with a significant reduction in cardiac index and mean arterial pressure and a significant increase in intracranial pressure, CRRT utilization has not. ${ }^{(22,23)}$ Consequently, CRRT was deemed safer than IRRT in patients with ALF and risk of cerebral edema. Therefore, CRRT use in patients with ALF appears to improve hyperammonemia and reduce the risk of cerebral edema and intracranial hypertension while avoiding hemodynamic changes that may exacerbate intracranial pressure.

Using only a specific threshold of serum ammonia to select patients for CRRT in this context might not be enough, given lack of sensitivity observed. Nevertheless, a serum ammonia level $\geq 150-200 \mu \mathrm{mol} / \mathrm{L}$ has been associated with increased risk of cerebral edema and intracranial hypertension. ${ }^{(15)}$ Although this study was not designed nor powered to determine a threshold serum ammonia value to stop CRRT, we would advocate for the decision to cease CRRT be based on further clinical data besides serum ammonia level (e.g., serum $\mathrm{pH}$, bicarbonate, and potassium, urine output, and fluid balance).

Taking into account pre-existent literature and our findings, we suggest that patients with ALF and hyperammonemia, especially those at greater risk for cerebral edema and intracranial hypertension (e.g., with highgrade $\mathrm{HE}$, need for vasopressors, or with acute kidney injury), should be considered for CRRT. ${ }^{(15,24)}$

Although serum ammonia modulation with CRRT may impact positively ALF patients' clinical outcomes, this becomes only one more adjunctive therapeutic strategy to control neurological failure. The timely treatment of all organ failures in the intensive care unit is of paramount importance and has contributed greatly for the overall improvement of these patients' clinical outcomes over the past decades. ${ }^{(6)}$

\section{LIMITATIONS}

This study had the following limitations that warrant consideration. First, this was a retrospective cohort and therefore may have been prone to selection bias. However, the large sample size, multicenter character, and the use of data from prospectively enrolled patients meeting entry criteria in the US-ALFSG registry may have helped to minimize the risk of such bias.

Second, further details on RRT specifics were not possible to obtain within the US-ALFSG registry. This additional data may have added to the explanation of RRT effect on ammonia dynamics and patients' clinical outcomes in ALF. Nevertheless, currently there is still controversy regarding most technical aspects of RRT use in general patients, including indications, early versus late initiation, intermittent versus continuous modes, diffusion versus convection modes, predilution versus postdilution fluid replacement, high versus low treatment dose, and stopping rules. ${ }^{(12,18)}$ For example, previous studies have shown that, among general critically ill patients, diffusion and convection modes have similar capacity for clearance of small molecules (e.g., urea, creatinine, ammonia, and glutamine). ${ }^{(25)}$ Furthermore, high $(\geq 35 \mathrm{~mL} / \mathrm{kg} / \mathrm{h})$ and low $(<35 \mathrm{~mL} / \mathrm{kg} / \mathrm{h})$ dose of renal replacement have been reported to have similar effect on mortality and renal function recovery. ${ }^{(26)}$ Even if, in specific subgroups of patients, these technical aspects may apply differently, the conclusions of such studies help to strengthen the validity of our results. ${ }^{(27)}$

Despite these limitations, we believe that our findings add to the previous literature on hyperammonemia and cerebral edema management in ALF. To date, this is the largest multicenter study to evaluate the effect of RRT on hyperammonemia in ALF. To further study the use of RRT in this context, clinical trials should be attempted in the near future. However, this may prove to be a difficult task; not only is ALF a rare disease, but also hyperammonemia may be difficult to isolate from classical indications for RRT initiation in the context of acute kidney injury. ${ }^{(12,27)}$

In a large cohort of patients with ALF, hyperammonemia was associated with worse high-grade $\mathrm{HE}$ and 21-day post study admission TFS. Furthermore, CRRT was associated with a reduction in serum ammonia level and improved 21-day post study admission TFS. In ALF, CRRT may be an effective adjunctive therapy to improve hyperammonemia as well as overall clinical outcomes.

\section{Appendix}

Members and institutions participating in the Acute Liver Failure Study Group 1998-2016 are as follows: W.M. Lee, M.D. (Principal Investigator); Anne M. 
Larson, M.D., Iris Liou, M.D., University of Washington, Seattle, WA; Oren Fix, M.D., Swedish Medical Center, Seattle, WA; Michael Schilsky, M.D., Yale University, New Haven, CT; Timothy McCashland, M.D., University of Nebraska, Omaha, NE; J. Eileen Hay, M.B.B.S., Mayo Clinic, Rochester, MN; Natalie Murray, M.D., Baylor University Medical Center, Dallas, TX; Obaid S. Shaikh, M.D., University of Pittsburgh, Pittsburgh, PA; Andres Blei, M.D., Northwestern University, Chicago, IL (deceased), Daniel Ganger, M.D., Northwestern University, Chicago, IL; Atif Zaman, M.D., University of Oregon, Portland, OR; Steven H.B. Han, M.D., University of California, Los Angeles, CA; Robert Fontana, M.D., University of Michigan, Ann Arbor, MI; Brendan McGuire, M.D., University of Alabama, Birmingham, AL; Raymond T. Chung, M.D., Massachusetts General Hospital, Boston, MA; Alastair Smith, M.B., Ch.B., Duke University Medical Center, Durham, NC; Robert Brown, M.D., Cornell/Columbia University, New York, NY; Jeffrey Crippin, M.D., Washington University, St Louis, MO; Edwin Harrison, M.D., Mayo Clinic, Scottsdale, AZ; Adrian Reuben, M.B.B.S., Medical University of South Carolina, Charleston, SC; Santiago Munoz, M.D., Albert Einstein Medical Center, Philadelphia, PA; Rajender Reddy, M.D., University of Pennsylvania, Philadelphia, PA; R. Todd Stravitz, M.D., Virginia Commonwealth University, Richmond, VA; Lorenzo Rossaro, M.D., University of California Davis, Sacramento, CA; Raj Satyanarayana, M.D., Mayo Clinic, Jacksonville, FL; Tarek Hassanein, M.D., University of California, San Diego, CA; Constantine J. Karvellas MD, University of Alberta, Edmonton, AB, Canada; Jodi Olson, M.D., University of Kansas, Kansas City, KS; Ram Subramanian, M.D., Emory, Atlanta, GA; James Hanje, M.D., Ohio State University, Columbus, OH; Bilal Hameed, M.D., University of California San Francisco, CA.

The University of Texas Southwestern Administrative Group included Grace Samuel, Ezmina Lalani, Carla Pezzia, and Corron Sanders, Ph.D., Nahid Attar, Linda S. Hynan, Ph.D., and the Medical University of South Carolina Data Coordination Unit included Valerie Durkalski, Ph.D., Wenle Zhao, Ph.D., Jaime Speiser, Catherine Dillon, Holly Battenhouse, and Michelle Gottfried.

\section{REFERENCES}

1) Wright G, Noiret L, Olde Damink SW, Jalan R. Interorgan ammonia metabolism in liver failure: the basis of current and future therapies. Liver Int 2011;31:163-175.
2) Clemmesen JO, Larsen FS, Kondrup J, Hansen BA, Ott P. Cerebral herniation in patients with acute liver failure is correlated with arterial ammonia concentration. HePATOLOGY 1999; 29:648-653.

3) Bernal W, Hall C, Karvellas CJ, Auzinger G, Sizer E, Wendon J. Encephalopathy and intracranial hypertension in acute liver failure. HePATOLOGY 2007;46:1844-1852.

4) Albrecht J, Norenberg MD. Glutamine: a Trojan horse in ammonia neurotoxicity. Hepatology 2006;44:788-794.

5) Butterworth RF. Pathogenesis of hepatic encephalopathy and brain edema in acute liver failure. J Clin Exp Hepatol 2015; 6(Suppl 1):S96-S103.

6) Bernal W, Hyyrylainen A, Gera A, Audimoolam VK, McPhail MJ, Auzinger G, et al. Lessons from look-back in acute liver failure? A single centre experience of 3300 patients. J Hepatol 2013;59:74-80.

7) Cordoba J, Blei AT, Mujais S. Determinants of ammonia clearance by hemodialysis. Artif Organs 1996;20:800-803.

8) Saliba F, Camus C, Durand F, Mathurin P, Letierce A, Delafosse B, et al. Albumin dialysis with a noncell artificial liver support device in patients with acute liver failure: a randomized, controlled trial. Ann Intern Med 2013;159:522-521.

9) Slack AJ, Auzinger G, Willars C, Dew T, Musto R, Corsilli D, et al. Ammonia clearance with haemofiltration in adults with liver disease. Liver Int 2014;34:42-48.

10) World Medical Association. Declaration of Helsinki. Ethical Principles for Medical Research Involving Human Subjects. JAMA 2013;310:2191-2194.

11) Nicolao F, Efrati C, Masini A, Merli M, Attili AF, Riggio O. Role of determination of partial pressure of ammonia in cirrhotic patients with and without hepatic encephalopathy. J Hepatol 2003;38:441-446.

12) Ronco C, Ricci Z, De Backer D, Kellum JA, Taccone FS, Joannidis $M$, et al. Renal replacement therapy in acute kidney injury: controversy and consensus. Crit Care 2015;19: 146.

13) Whitley E, Ball J. Statistics review 4: sample size calculations. Crit Care 2002;6:335-341.

14) Bhatia V, Singh R, Acharya SK. Predictive value of arterial ammonia for complications and outcome in acute liver failure. Gut 2006;55:98-104.

15) Bernal W, Hall C, Karvellas CJ, Auzinger G, Sizer E, Wendon J. Arterial ammonia and clinical risk factors for encephalopathy and intracranial hypertension in acute liver failure. HEPATOLOGY 2007;46:1844-1852.

16) Bernal W, Auzinger G, Dhawan J, Wendon J. Acute liver failure. Lancet 2010;376:190-201.

17) Bernal W, Wendon J. Acute liver failure. N Eng J Med 2013; 369:2525-2534.

18) Karvellas CJ, Farhat MR, Sajjad I, Mogensen SS, Leung AA, Wald $\mathrm{R}$, et al. A comparison of early versus late initiation of renal replacement therapy in critically ill patients with acute kidney injury: a systematic review and meta-analysis. Crit Care 2011;15:R72.

19) Leite TT, Macedo E, Pereira SM, Bandeira SR, Pontes PH, Garcia AS, et al. Timing of renal replacement therapy initiation by AKIN classification system. Crit Care 2013;17:R62.

20) Uchino S, Fealy N, Baldwin I, Morimatsu H, Bellomo R. Continuous is not continuous: the incidence and impact of circuit "down-time" on uraemic control during continuous venovenous haemofiltration. Intensive Care Med 2003;29:575578. 
21) Larsen FS, Schmidt LE, Bernsmeier C, Rasmussen A, Isoniemi $\mathrm{H}$, Patel VC, et al. High-volume plasma exchange in patients with acute liver failure: an open randomised controlled trial. J Hepatol 2016;64:69-78.

22) Davenport A, Will EJ, Davison AM. Effect of renal replacement therapy on patients with combined acute renal and fulminant hepatic failure. Kidney Int Suppl 1993;41:S245-51.

23) Davenport A, Will EJ, Davison AM. Improved cardiovascular stability during continuous modes of renal replacement therapy in critically ill patients with acute hepatic and renal failure. Crit Care Med 1993;21:328-338.

24) Tujios SR, Hynan LS, Vazquez MA, Larson AM, Seremba E, Sanders CM, et al. Clin Gastroenterol Hep 2015;13:352359.
25) Ricci Z, Ronco C, Bachetoni A, D'amico G, Rossi S, Alessandri $\mathrm{E}$, et al. Solute removal during continuous renal replacement therapy in critically ill patients: convection versus diffusion. Crit Care 2006;10:R67.

26) Fayad AI, Buamscha DG, Ciapponi A. Intensity of continuous renal replacement therapy for acute kidney injury. Cochrane Database Syst Rev 2016;10:CD010613.

27) Hoste EA, Dhondt A. Clinical review: use of renal replacement therapies in special groups of ICU patients. Crit Care 2012;16:201.

\section{Supporting Information}

Additional Supporting Information may be found at onlinelibrary.wiley.com/doi/10.1002/hep.29488/suppinfo. 